\title{
On the Behavior of Positrons in CdTe under Compression
}

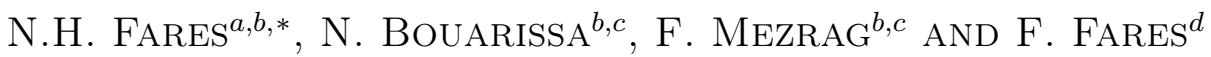 \\ ${ }^{a}$ Department of Informatics, Faculty of Computer Science and Mathematics, \\ University of Bordj-Bou-Arréridj, 34000, Algeria \\ ${ }^{b}$ Laboratory of Materials Physics and Its Applications, University of M'sila, 28000 M'sila, Algeria \\ ${ }^{c}$ Physics Department, Faculty of Science, University of M'sila, 28000 M'sila, Algeria \\ ${ }^{d}$ Electronics Department, Faculty of Science, University Bordj-Bou-Arréridj, 34000, Algeria
}

\begin{abstract}
We report on the positron chemical potential, positron band effective mass, positron affinity, and positron bulk lifetime in cadmium telluride under compression. The calculations are carried out using a pseudo-potential approach. The ionic potential is treated using the point-core approximation. At zero pressure, our results agree reasonably well with those reported in the literature. Upon compression, the positron characteristics vary monotonically indicating that the positron annihilates differently in CdTe when pressure is applied. The present study should provide essential information in the positron annihilation in CdTe under pressure.
\end{abstract}

DOI: 10.12693/APhysPolA.137.502

PACS/topics: positron, CdTe, high-pressure

\section{Introduction}

II-V compound semiconductors have attracted much attention during the last years $[1-12]$. This is due to their potential use for technological applications, which ranges from optoelectronic devices to luminescence biological tags. CdTe belongs to this family of materials and crystallizes in the zinc-blende structure. The material in question found a use as an infrared optical material [13] and is considered as a suitable absorber layer for $\mathrm{CdTe} / \mathrm{CdS}$ solar cells devices [14-16].

The study of materials under high-pressure becomes an important field for investigations. This is mainly due to the development of the diamond anvil cell technology and to the advancement of computational techniques and new technological methods [17-26]. This has allowed for investigation of several fundamental properties of semiconducting materials under high-pressure.

Positron annihilation spectroscopy and slow-positronbeam technique are powerful tools for studying bulk and solid surfaces $[27,28]$. On the other hand, the theoretical developments using the Monte Carlo simulation techniques and other methods have allowed for a satisfactory analysis and interpretation of experimental results [29-37].

In this contribution, the positron behavior under highpressure in CdTe has been investigated. Features such as positron chemical potential affinity, and bulk lifetime are reported and their dependence on high-pressure are examined and discussed.

*corresponding author; e-mail: fares_houda@yahoo.fr

\section{Computations}

The electron wave functions have been calculated using the empirical pseudopotential method (EPM) [38, 39], where the empirical pseudopotential parameters are optimized using the non-linear least-squares method, as described in Refs. [40, 41]. Table I shows the energy bandgaps fixed with the fits for CdTe at zero pressure.

\section{TABLE I}

Experimental band-gap energies for CdTe fixed in the fits $[42,43]$

\begin{tabular}{c|c|c|c}
\hline \hline Compound & $E_{\Gamma-\Gamma}[\mathrm{eV}]$ & $E_{\Gamma-X}[\mathrm{eV}]$ & $E_{\Gamma-L}[\mathrm{eV}]$ \\
\hline $\mathrm{CdTe}$ & 1.5045 & 3.48 & 2.47
\end{tabular}

The positron wave functions are described by plane waves and the ionic potential is treated using the pointcore approximation. The wave function of the fully thermalized positron is considered in this work. More details about the approach used were reported in Ref. [44].

The electron and positron chemical potentials are obtained following the approach of Boev et al. [45], whereas the positron bulk lifetime is calculated using the Siethoff relation [46].

For pressures larger than zero, we used a procedure similar to that used in Ref. [47] for obtaining the pseudopotential form factors. The lattice parameter is determined using the Murnaghan equation of state. The input data are those reported in Ref. [47] for CdTe.

\section{Results and discussion}

The positron chemical potential $\mu_{+}$is calculated from the positron band structure. It corresponds to the lowest energy in the band. At zero pressure, $\mu_{+}$is found to be $-7.5408 \mathrm{eV}$ for positron in CdTe. The variation of $\mu_{+}$as 


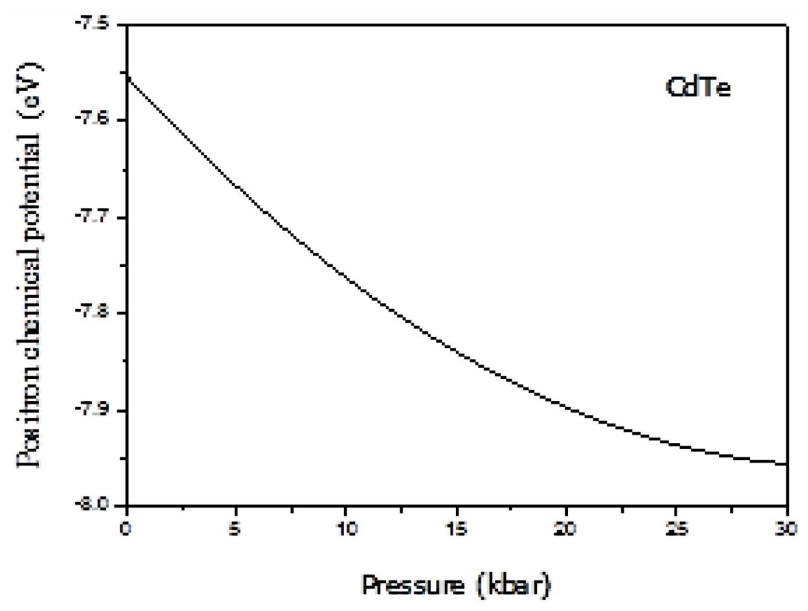

Fig. 1. Positron chemical potential in CdTe versus pressure.

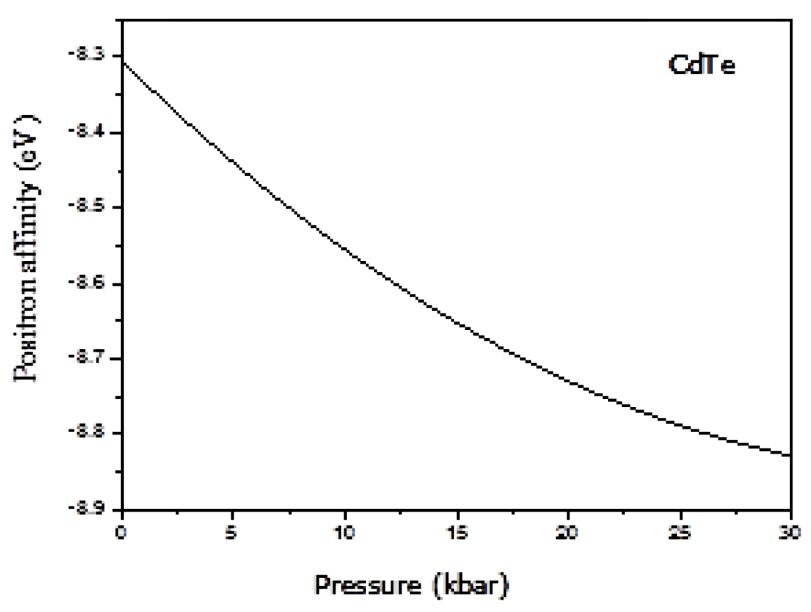

Fig. 2. Positron affinity in CdTe versus pressure.

a function of pressure is displayed in Fig. 1. Note that as pressure increases up to $30 \mathrm{kbar}, \mu_{+}$decreases monotonically and non-linearly. The behavior of $\mu_{+}$versus pressure can be attributed to the decrease of the lattice parameter under applied pressure which augments the number of scattering, leading thus to the diminution of the positron thermalization energy.

The positron affinity $A_{+}$is an important parameter in the understanding of positron trapping at interfaces and precipitates $[34,48]$. It is linked to both electron and positron bands. $A_{+}$has been calculated in this work using the expression $[34,49]$ :

$$
A_{+}=\mu_{-}+\mu_{+} \text {. }
$$

where $\mu$ is the electron chemical potential.

At zero pressure, we predict a value of $A_{+}=-8.29 \mathrm{eV}$ for positron in CdTe. The variation of $A_{+}$as a function of pressure is plotted in Fig. 2. By observing Fig. 2 one can note that $A_{+}$decreases monotonically and non-linearly with pressure raising up to 30 kbar. The diminution

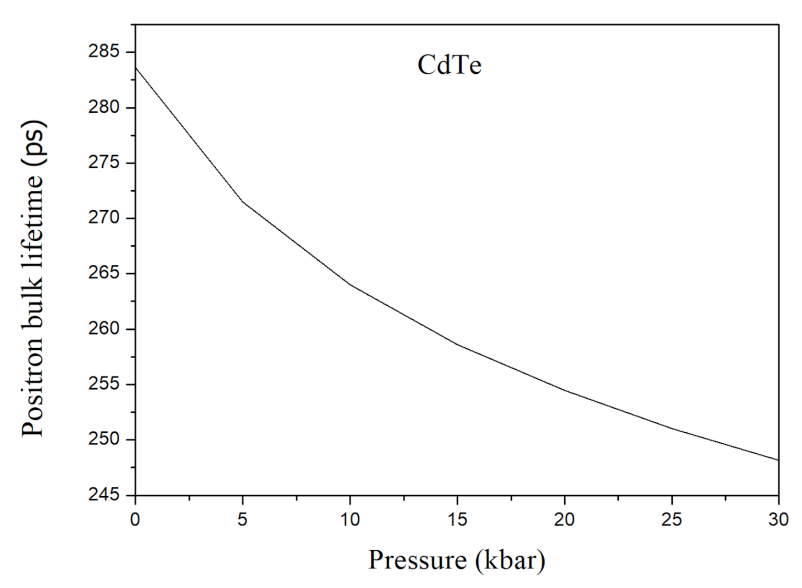

Fig. 3. Positron bulk lifetime in CdTe versus pressure.

of $A_{+}$upon compression can be traced back to the positron migration from the interstitial region to the inter-nuclear spacing. This suggests that the positron in CdTe under compression annihilates differently from the positron in CdTe at zero pressure.

Another interesting parameter in the investigation of positron annihilation is the positron bulk lifetime. The latter has been calculated using the Siethoff relation [46]. Our findings yielded a value of 283.66 ps for positron in CdTe at zero pressure. Barbiellini et al. [50] have calculated positron bulk lifetime for zinc-blende CdTe. Their results yielded values of 228 ps (using LMTO-ASA within the LDA), 290 ps (using LMTO ASA within the GGA), 228 ps (using the atomic superposition within the LDA), and $310 \mathrm{ps}$ (using the atomic superposition within the GGA). Our result appears to be in better agreement with the GGA calculations as compared to the LDA ones.

The pressure dependence of the positron bulk lifetime in CdTe is illustrated in Fig. 3. Note that as pressure is enhanced from 0 to $30 \mathrm{kbar}$, the positron bulk lifetime decreases almost linearly. In their recent paper, Fares and Bouarissa [51] have linked the trend of the positron bulk lifetime to that of the crystal ionicity in a given semiconductor material. Accordingly, we may expect that the ionicity of CdTe decreases with enhancing pressure. This is consistent with previous studies of the behavior of ionicity in semiconductors under high-pressure [52].

\section{Conclusion}

Based essentially on the EPM, the positron characteristics in CdTe under pressure were investigated. The pressure dependence of the positron chemical potential, affinity, and bulk lifetime was examined and discussed while highlighting a decrease of all features of interest when pressure is applied. These findings indicate that the positron annihilates differently upon compression. The parameters predicted in this contribution can serve as a support for future experiments. 


\section{References}

[1] II-VI Semiconductor Compounds (University Western Australia), Ed. M. Jain, World Sci., Singapore 1993.

[2] in: Handbook on Physical Properties of semiconductors, II-VI Compound Semiconductors, Vol. 3, Ed. S. Adachi, Springer, US 2004.

[3] S. Saib, N. Bouarissa, Phys. Status Solidi B 244, 1063 (2007).

[4] M. Isshiki, J. Wang, in: Springer Handbook of Electronic and Photonic Materials, Springer Handbooks, Eds. S. Kasap, P. Copper, Springer, Cham 2007, p. 1-1.

[5] L. Hannachi, N. Bouarissa, Physica B 404, 3650 (2009).

[6] A. Gueddim, N. Bouarissa, A. Villesuzanne, Phys. Scr. 80, 055702 (2009).

[7] S. Coe, W.K. Woo, M. Bawendi, V. Bulović, Nature 420, 800 (2002).

[8] N. Tessler, V. Medvedev, M. Kazes, S. Kan, U. Banin, Science 295, 1506 (2002).

[9] D. Gal, G. Hodes, Appl. Phys. Lett. 73, 3135 (1998).

[10] S. Saib, N. Bouarissa, P. Rodríguez-Hernández, A. Muñoz, Eur. Phys. J. B 73, 185 (2010).

[11] Wide Band Gap Semiconductors, Ed. C.G. Van de Walle, North-Holland, Amsterdam 1993.

[12] J.K. Jaiswal, H. Mattoussi, J.M. Mauro, S.M. Simon, Nat. Biotech. 21, 47 (2003).

[13] D.G. Harris, Infrared Phys. Technol. 39, 185 (1998).

[14] A. Luqye, S. Hegedus, Handbook of Photovoltaic Science and Engineering, Wiley, 2009.

[15] X. Wu, Sol. Energy 77, 803 (2004).

[16] M.F. Hasaneen, W.S. Mohamed, Optik 160, 307 (2018).

[17] W.B. Holzapfel, Rep. Prog. Phys. 59, 29 (1996) and references therein.

[18] G.J. Ackland, Rep. Progr. Phys. 64, 483 (2001) and references therein.

[19] N. Bouarissa, Phys. Status Solidi B 231, 391 (2002).

[20] S. Saib, N. Bouarissa, P. Rodríguez-Hernández, A. Muñoz, Physica B 403, 4059 (2008).

[21] A. Mujica, A. Rubio, A. Muñoz, R.J. Needs, Rev Mod. Phys. 75, 863 (2003) and references therein.

[22] N. Bouarissa, Mater. Chem. Phys. 73, 51 (2002).

[23] K. Daviau, K.K.M. Lee, Crystals 8, 217 (2018).

[24] M. Miao, W.R. Lambrecht, Phys. Rev. Lett. 94, 225501 (2005).

[25] N. Bouarissa, Physica B 406, 2583 (2011).

[26] S. Daoud, N. Bioud, N. Bouarissa, Mater. Sci. Semicond. Process. 31, 124 (2015).
[27] P.J. Schultz, K.G. Lynn, Rev. Mod. Phys. 60, 701 (1988).

[28] Positron Beams and Their Applications, Ed. P.G. Coleman, World Sci., Singapore 2000.

[29] F. Tuomisto, I. Makkonen, Rev. Mod. Phys. 85, 1583 (2013).

[30] N. Bouarissa, A.B. Walker, H. Aourag, J. Appl. Phys. 83, 364 (1998).

[31] M. Dapor, A. Miotello, Scann. Microsc. 12, 131 (1998).

[32] M. Dapor, J. Electron. Spectosc. Relat. Phenom. 151, 182 (2006)

[33] N. Bouarissa, Philos. Mag. B 80, 1743 (2000).

[35] I. Makkonen, A. Snicker, M.J. Puska, J.M. Maki, F. Tuomisto, Phys. Rev. B 82, 041307 (2010).

[36] N. Bouarissa, J. Phys. Chem. Solids 67, 1440 (2006).

[37] A. Zubiaga, F. Tuomisto, M.J. Puska, Phys. Rev. A 89, 052707 (2014).

[38] M.L. Cohan, J.R. Chelikowsky, Electronic Structure and Optical Properties of Semiconductors, Springer, Berlin 1989.

[39] N. Bouarissa, Mater. Sci. Eng. B 86, 53 (2001).

[40] T. Kobayasi, H. Nara, Bull. Coll. Med. Sci. Tohoku Univ. 2, 7 (1993).

[41] N. Bouarissa, M. Boucenna, Phys. Scr. 79, 015701 (2009).

[42] J. Yang, F. Zidou, T. Shapira, J. Appl. Phys. 91, 703 (2002).

[43] A. Kobayashi, O.F. Sankey, J.D. Dow, Phys. Rev. B 25, 6367 (1982).

[44] N. Bouarissa, J. Phys. Chem. Solids 61, 109 (2000).

[45] O.V. Boev, M.J. Puska, R.M. Nieminen, Phys. Rev. B 36, 7786 (1987)

[46] H. Siethoff, Phys. Status Solidi B 205, 1948 (1998).

[47] N. Bouarissa, S. Saib, M. Boucenna, F. Mezrag, Comput. Condens. Matter 17, e00346 (2018).

[48] J. Kuriplach, M. Job, G. Brauer, W. Arwand, E.M. Nielet, P.G. Coleman, N. Wangner, Phys. Rev. $B$ 59, 1948 (1999).

[49] M.J. Puska, P. Lanki, R.M. Nieminen, J. Phys. Condens. Matter 1, 6081 (1989).

[50] B. Barbiellini, M.J. Puska, T. Korhonen, A. Harju, T. Torsi, R.M. Nieminen, Phys. Rev. B 53, 16201 (1996).

[51] N.E.H. Fares, N. Bouarissa, J. Electron. Spectrosc. Relat. Phenom. 206, 1 (2016).

[52] M. Boucenna, N. Bouarissa, Mater. Chem. Phys. 84, 375 (2004). 